Immerhin steht diese minimale Restschwingung wenigstens dem Sinne nach in guter Übereinstimmung mit.den Zahlen, die ich ${ }^{1}$ ) aus den Pulkowaer Beobachtungen des Zenitsterns $\delta$ Cassiopeiae für die Tagesschwankung von $\varphi$ abgeleitet habe, indem auch diese am Tage positive, in der Nacht negative Zeichen aufweisen. Es dürfte aber kaum einem $Z$ weifel unterliegen, daß eine solche Welle in den Beobachtungen, wenn sie überhaupt reell ist, nicht einer Berlin-Babelsberg, I920 Dez. I 3 .
1) AN 207.3.
2) AN 169.II3.
3) AN 193.347.
4) AN 207.I.

tatsächlichen Veränderlichkeit der Polhöhe entspringt, sondern ihren natürlichen Grund in Saalrefraktion hat.

Abschließend läßt sich also sagen, daß in völliger Bestätigung der Ergebnisse früherer Beobachtungen und Untersuchungen von Kimur $^{2}$ ), Wanach $^{3}$ ) und mir ${ }^{4}$ ), auch aus dem neuen Boccardischen Beobachtungsmaterial eine praktisch irgendwie merkliche regelmäßige Schwankung der Polhöhe mit der Periode eines mittleren Sonnentages $\mathrm{ni}$ ch $\mathrm{t}$ nachzuweisen ist.

\title{
Helligkeitsmessungen veränderlicher Sterne und der Nova Aquilae 3 (1918).
}

$\mathrm{X}$ Aurigae sollte im Jahre t920 nach Hartwigs Katalog am 29. April und $\mathrm{R} C$ anum venaticorum am 24. Juni ein Maximum haben. Wie die folgenden Helligkeitsmessungen zeigen, traten beide Maxima bedeutend später ein. Es gibt die erste Tabelle die Größenklassendifferenzen zwischen $\mathrm{X}$ Aurigae und einem Vergleichstern, welcher nach Graff

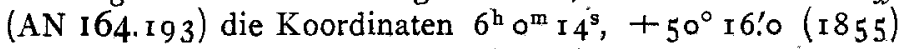
und die Helligkeit $10^{\mathrm{m}} 25$ besitzt, die $z$ weite Tabelle die Größenklassendifferenzen $z$ wischen $R$ Canum venaticorum und dem Vergleichstern $\mathrm{BD}+40^{\circ} 2695$.

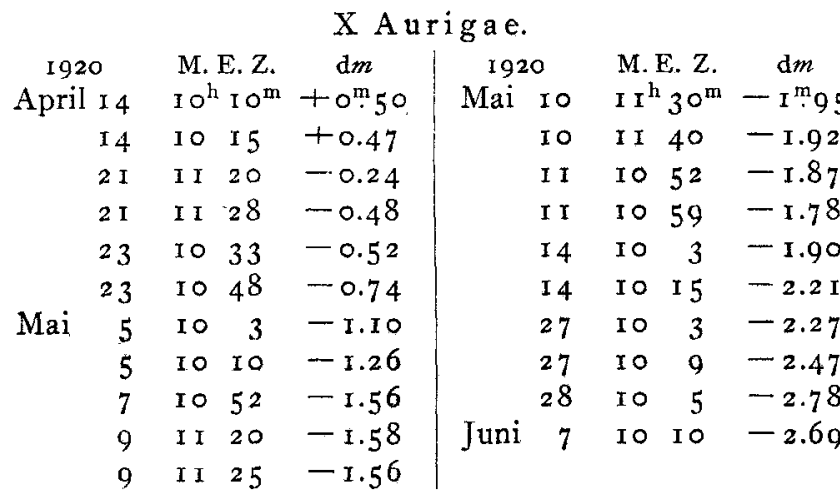

R Canumvenaticorum.

\begin{tabular}{|c|c|c|c|c|c|c|c|c|}
\hline \multirow{2}{*}{\multicolumn{2}{|c|}{$\begin{array}{c}\text { 1920 } \\
\text { April i4 }\end{array}$}} & \multirow{2}{*}{\multicolumn{2}{|c|}{ M. E. Z. }} & \multirow{2}{*}{$\begin{aligned} & \mathrm{d} m \\
+ & \mathrm{o}^{\mathrm{m}} \mathrm{I} 4\end{aligned}$} & \multicolumn{2}{|c|}{1920} & M. E. 2 . & \multirow{2}{*}{$\begin{array}{c}\mathrm{d} m \\
-0^{\mathrm{m}} \cdot 96\end{array}$} \\
\hline & & & & & Mai & 25 & I $2^{h} 44^{\mathrm{m}}$ & \\
\hline & $2 I$ & $I_{4}$ & 8 & +0.13 & & 28 & ro 35 & -1.29 \\
\hline \multirow[t]{8}{*}{ Mai } & 5 & 10 & $5^{\circ}$ & $-0.6 \mathrm{r}$ & Juni & 7 & I 2 & $-\mathrm{I} .27$ \\
\hline & 7 & I I & 32 & -0.60 & & 8 & ro $5^{x}$ & $-1.3^{2}$ \\
\hline & 7 & I I & 42 & -0.55 & & 8 & II 16 & -1.32 \\
\hline & 9 & 12 & $3^{I}$ & -0.76 & & 9 & I $242^{\circ}$ & - I.49 \\
\hline & 9 & I 2 & 44 & -0.62 & & 9 & I 3 & $-\mathrm{I} .68$ \\
\hline & 10 & is & 3 & -0.64 & & I I & I 156 & $-\mathrm{I} .64$ \\
\hline & I I & I 2 & 58 & -0.72 & & I I & 12 & $-I .52$ \\
\hline & I I & 13 & 4 & -0.77 & & 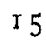 & I I 55 & - I. 64 \\
\hline
\end{tabular}
Juni 15 M. E. Z

$$
\begin{array}{r}
10 \\
14
\end{array}
$$$$
\text { Juli } \begin{array}{rrrr}
24 & \text { I0 } & 35 & - \text { I.90 } \\
6 & \text { IO } & 2 \mathrm{I} & -\mathrm{I} .9 \mathrm{I} \\
6 & \text { IO } & 3 \mathrm{I} & -\mathrm{I} .9 \mathrm{I} \\
\mathrm{IO} & \text { II } & 5 & -\mathrm{I} .79 \\
\text { IO } & \text { I I } & \text { I } 5 & -\mathrm{I} .73
\end{array}
$$$$
\text { I I I I } 3-2.06
$$

Königstuhl, I 920 Sept. 24.

$$
\begin{aligned}
& \begin{array}{ccc}
\text { I920 } & \text { M.E. Z. } & \text { d } m \\
\text { Juli I I } & \text { I } 2^{\mathrm{h}} 23^{\mathrm{m}} & -2^{\mathrm{m}} \cdot \mathrm{I} 4
\end{array} \\
& \text { I } 4 \text { II } 4-\text { I.76 } \\
& \text { I } 5 \text { II } \circ-1.78 \\
& \begin{array}{llll}
I 7 & \text { I } 5 I & -1.67
\end{array} \\
& \text { I } 7 \text { I } 2 \text { I }-I .55 \\
& 2 \mathrm{I} \quad \mathrm{I} \text { I } 7-\mathrm{I} .79 \\
& \begin{array}{llll}
21 & 127 & -1.74
\end{array} \\
& \text { Aug. } 7 \quad 948-\text { I.I } 4 \\
& 7 \text { IO IO }- \text { I.I } 3
\end{aligned}
$$

\begin{tabular}{|c|c|c|c|c|c|c|c|c|c|}
\hline & \multicolumn{2}{|c|}{ M. E. Z. } & \multirow{2}{*}{$\begin{array}{l}N-V \\
0.5 I\end{array}$} & \multicolumn{2}{|c|}{1920} & \multicolumn{2}{|c|}{ M. E. Z. } & \multirow{2}{*}{$\begin{array}{l}N-V \\
O^{m} \cdot 58\end{array}$} \\
\hline Juni & 7 & & $49^{\mathrm{m}}$ & & Juli & 6 & $10^{h}$ & $47^{\mathrm{m}}$ & \\
\hline & 8 & I. I & 39 & 0.62 & & ro & I I & 22 & 0.66 \\
\hline & 8 & I I & 55 & 0.65 & & 10 & I I & $3^{2}$ & 0.59 \\
\hline & 9 & I I & 54 & 0.66 & & I I & 12 & 34 & 0.48 \\
\hline & 9 & I 2 & 6 & 0.54 & & I I & 12 & 47 & $0.5 \mathrm{I}$ \\
\hline & I I & I 3 & 9 & $0.5^{2}$ & & I 4 & I I & 30 & 0.33 \\
\hline & 12 & 14 & 18 & $0.2 \mathrm{I}$ & & I 5 & I I & $5^{I}$ & $0.5 I$ \\
\hline & I 2 & 14 & 25 & $0.3 \mathrm{I}$ & & I 5 & I 2 & I & 0.42 \\
\hline & I 5 & I I & 40 & 0.46 & & I 7 & I 2 & 28 & 0.65 \\
\hline & I 5 & II & 50 & 0.49 & & I 7 & I 3 & I 2 & 0.47 \\
\hline & I 6 & I 2 & 35 & 0.55 & & I 9 & İ I & I 5 & 0.39 \\
\hline & 19 & 12 & 22 & 0.46 & & I 9 & I I & 27 & $0.3^{8}$ \\
\hline & I 9 & I 2 & 33 & 0.35 & & 20 & I I & I I & 0.43 \\
\hline & 24 & I 2 & 7 & 0.68 & & 20 & I I & 20 & 0.44 \\
\hline & 24 & 12 & I 8 & 0.59 & & $2 I$ & I 2 & 40 & $0.3^{6}$ \\
\hline Juli & 6 & ro & $4 \mathrm{I}$ & 0.67 & & $2 \mathrm{I}$ & I 2 & $5^{2}$ & 0.40 \\
\hline
\end{tabular}

Nova Aquilae 3 ( 1918 ).

Die folgende Messungsreihe bildet die Fortsetzung zu der in den $A N 210.3^{83}$. Unter $\mathrm{N}-\mathrm{V}$ sind die Helligkeitsdifferenzen zwischen der Nova und dem Vergleichstern BD $+0_{4023}$ gegeben. Diese zeigen, daß auch im Juni und Juli dieses Jahres noch Helligkeitsschwankungen mit einer Periode von 7-8 Tagen stattfanden.

\section{Meteorfall im südlichen Holstein.}

Einer meiner kleinen, zehnjährigen Schüler erzählte von einer Erscheinung, die er an einem Apriltage I92 I gesehen hatte und die augenscheinlich ein Meteorfall war. Er hatte einen rotglühenden Gegenstand aus fast zenitaler, nur wenig nördlicher Richtung auf die Schnelsener Straße Voskamp auf etwa $100 \mathrm{~m}$ Entfernung von seinem Standorte aufschlagen gesehn. Das wäre nach schwachem Sausen oder Zischen mit einem dumpfen Knall geschehen. Er wäre sogleich nach der Stelle gelaufen und hätte dort einen schwärzlichen, schlackenähnlichen Stein vorgefunden. Er hätte ihn aber liegen gelassen, weil er zu heiß gewesen wäre. Der kleine Schüler, Albert Adler-Henning, hatte leider versäumt, mir sofort Mitteilung zu machen. Erst am $x 8$. April I 92 I erzählte er davon, nachdem mehr als eine Woche verstrichen war. So ließ sich 